\title{
Treatment of paroxysmal nodal tachycardia by dual demand pacemaker in the coronary sinus
}

\author{
D B O'KEEFFE, P V L CURRY, E SOWTON \\ From the Department of Cardiology, Guy's Hospital, London
}

SUMMARY A patient with refractory paroxysmal atrioventricular nodal re-entrant tachycardia had required direct current cardioversion to terminate attacks on 83 occasions. A dual demand pacemaker was implanted to sense and interrupt attacks of tachycardia automatically. The pacing electrode was positioned in the proximal coronary sinus near to the atrioventricular node; a site from which fixed rate underdrive pacing successfully interrupted attacks throughout a trial period of one week, with a lead left in this position on a temporary basis. Complete control of the arrhythmia was obtained in the six months after pacemaker implantation.

Paroxysmal re-entrant supraventricular tachycardia may prove refractory to optimum antiarrhythmic drug therapy, despite guidance of the latter by intracardiac electrophysiological studies. An alternative form of management for such cases is permanent pacing to sense and interrupt attacks as they arise. ${ }^{1-3}$ Recently such pacemaker systems have been made to work automatically, ${ }^{4}$ but have only been used for patients with paroxysmal re-entrant tachycardias associated with the Wolff-Parkinson-White syndrome. In such cases the anatomical circuit is large and pacing electrodes can easily be placed near the circuit for optimum efficiency of the system via the transvenous route. In atrioventricular nodal reentrant tachycardia, the anatomical circuit is extremely small and poses practical difficulties in terms of optimum placement of the tip of the pacemaker lead.

We report a case of refractory paroxysmal reentrant atrioventricular nodal tachycardia successfully treated by permanent dual demand pacing, with the pacing lead located in the coronary sinus and with the electrode at the ostium of the coronary sinus, a point close to the atrioventricular node.

\section{Case report}

A 67-year-old woman was referred with a 12-year history of recurrent attacks of paroxysmal supraventricular tachycardia that were becoming both more severe and more frequent. The optimum use of antiarrhythmic drugs on an empirical basis failed to provide adequate prophylaxis: such drugs had included digoxin, verapamil, beta-blocking drugs, disopyramide, quinidine, and procainamide. She had undergone direct current cardioversion on 83 occasions.

Apart from a soft systolic murmur audible at the apex of the heart, examination of the cardiovascular system was normal. Chest $x$-ray showed a normal heart size and configuration and both echocardiogram and thyroid function tests were normal.

A resting surface electrocardiogram showed sinus rhythm with notched $P$ waves in leads II and V2, and no evidence of pre-excitation (PR interval $0.14 \mathrm{~s}$ ). During paroxysmal tachycardia, the electrocardiogram showed regular narrow $Q R S$ complexes at a rate of 190 beats a minute with a QRS vector unchanged from that in sinus rhythm (Fig. 1). Discrete $P$ wave activity could not be distinguished on this tracing. An intracardiac electrophysiological study $^{5}$ performed in November 1978 showed that the supraventricular tachycardia was re-entrant in mechanism and that the circuit was located within the atrioventricular node. There was no evidence of the Wolff-Parkinson-White syndrome. Attacks could easily be initiated over a wide zone by timed premature extrastimuli introduced in the right atrium, as well as by incremental atrial pacing. During established attacks the heart rate was 175 beats a minute; considerably less than the maximum heart rate that could be tolerated on a $1: 1$ basis by the two atrioventricular pathways comprising the 


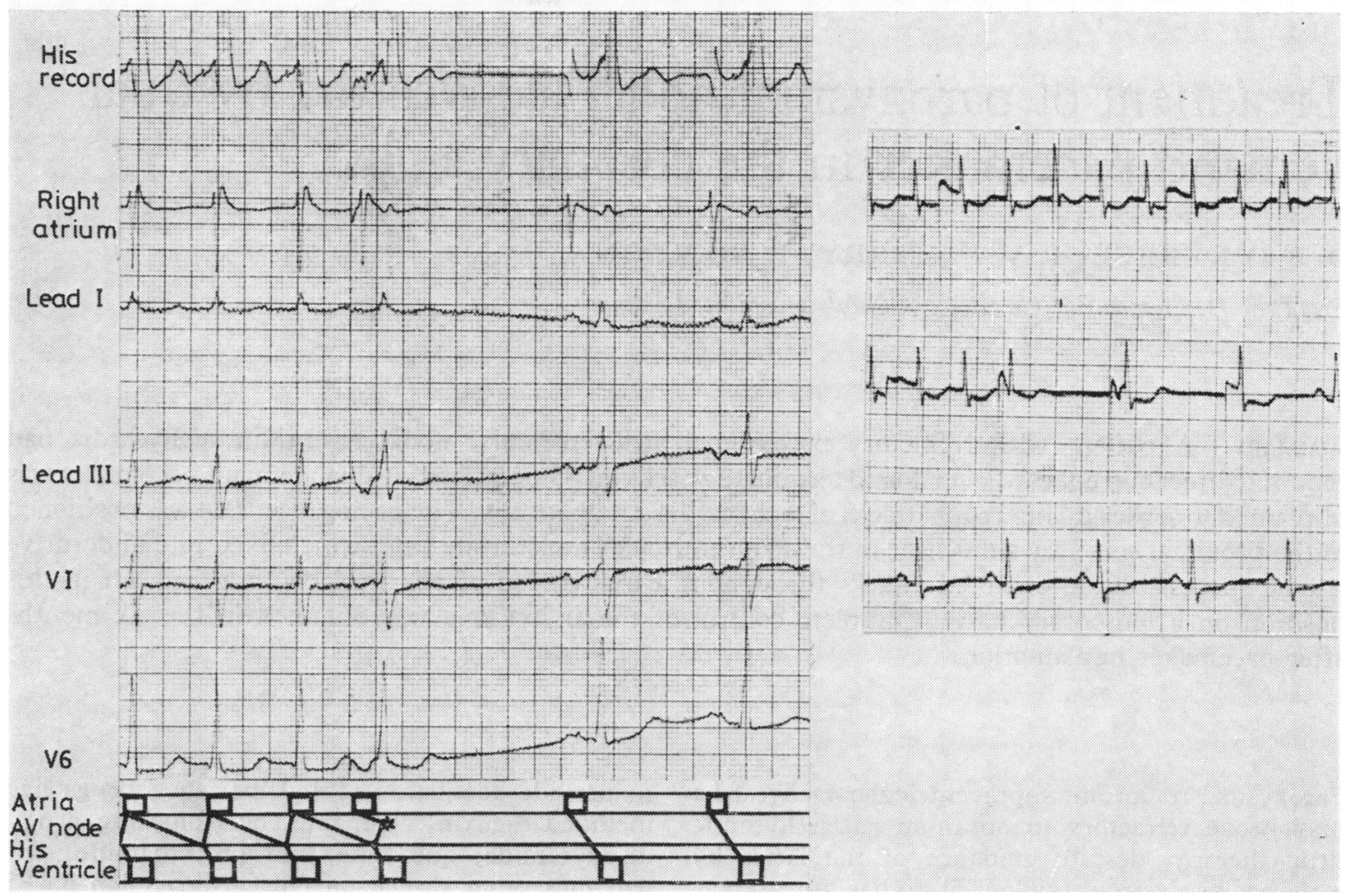

Fig. 1 Left-hand panel, showing termination of a reciprocating atrioventricular nodal tachycardia by a single timed extrastimulus delivered in the proximal coronary sinus.

Right-hand panel, surface electrocardiogram, lead II, three strips in sequence showing termination of tachycardia by the implanted dual demand pacemaker with the active electrode in the coronary sinus.

circuit (the Wenckebach phenomenon occurred anterograde at 230 beats a minute and retrograde at 240 beats a minute). The tachycardia could be reproducibly terminated by a single timed premature extrastimulus from either low right atrium or proximal coronary sinus but not from the right ventricle (Fig. 1). Competitive fixed-rate underdrive pacing at 70 beats a minute from the proximal coronary sinus site consistently terminated the tachycardia with an average delay of 26 seconds (range two seconds to five minutes). A temporary bipolar pacing wire was left in situ in the coronary sinus after the acute electrophysiological study. During the subsequent week when attacks occurred spontaneously or were initiated from this temporary electrode, fixed rate pacing at 70 beats a minute successfully and reliably interrupted attacks, with delays ranging from less than five seconds to 15 minutes (Fig. 2).

PERMANENT DUAL-DEMAND PACING In view of the success of the temporary external underdrive pacing system in controlling the tachycardia, the patient was fitted with an implantable unit (Telectronics $150 \mathrm{~B}$ custom) with dual demand characteristics such that it went into fixed rate mode at 70 beats a minute on sensing intrinsic heart rates below 70 beats a minute or above 150 beats a minute.

This unit was attached to a Cordis 324-788 unipolar coronary sinus lead (which has a $6 \mathrm{~cm}$ insulated tail on the distal end) positioned in the coronary sinus so that the electrode lies close to the coronary sinus ostium (Fig. 3), that is to say, as close as practicable to the atrioventricular node.

This system was shown to be able to terminate tachycardia (Fig. 1) and the pulse generator was implanted in a left pectoral subcutaneous pocket.

\section{FOLLOW-UP DATA}

The patient was given verapamil $40 \mathrm{mg}$ three times daily (though this had proved ineffective prophylaxis before insertion of the pacemaker) on the theoretical basis that by introducing delay in the anterograde pathway the "window" for termination 


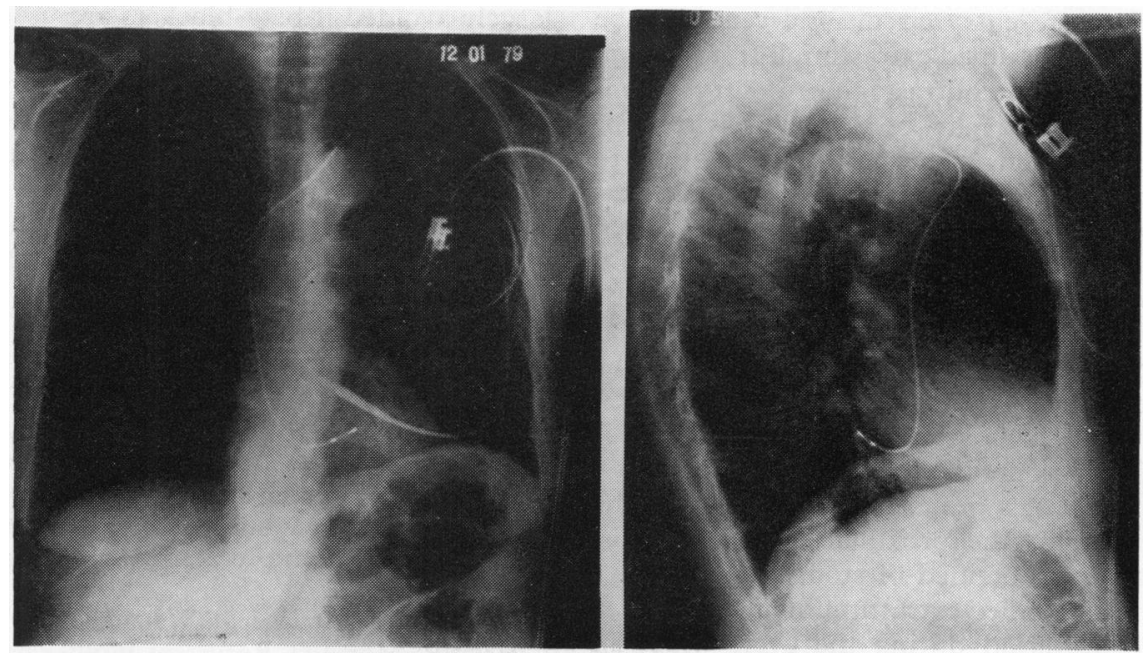

Fig. 2 Chest $\mathrm{x}$-ray showing the position of the temporary bipolar pacing wire in the proximal coronary sinus. This system was left in position for one week to test its efficacy under ambulant conditions.

of the tachycardia would increase in duration. Since the underdrive pacing impulses fall in a random fashion during the tachycardia, this would reduce the mean time taken to terminate tachycardia.

After six months of follow-up, the patient had become aware of tachycardia beginning on numerous occasions and was aware that the arrhythmia had been terminated after a variable period, usually from a few seconds to a few minutes. On a single occasion the arrhythmia reportedly persisted for two hours before terminating. On no occasion since implantation of the dual demand pacemaker has acute drug treatment or direct current cardioversion been required.

\section{Discussion}

Paroxysmal atrioventricular tachycardias associated with the Wolff-Parkinson-White syndrome involve

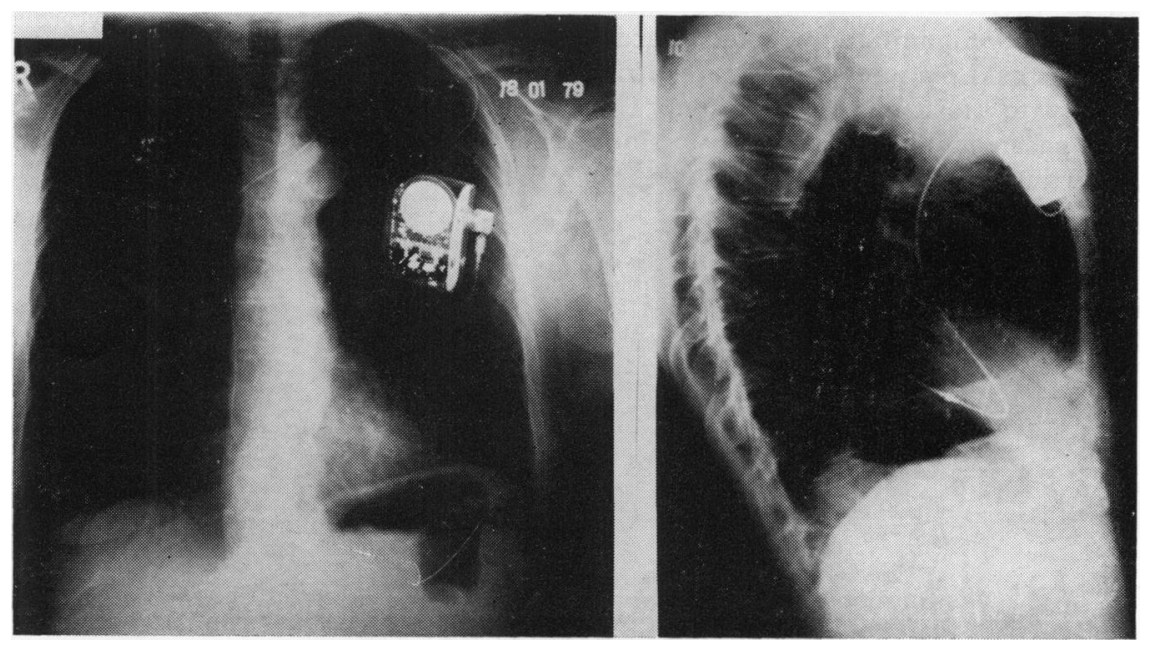

Fig. 3 Chest $\mathrm{x}$-ray showing the position of the implanted permanent pacing system. The electrode of the unipolar pacing catheter lies in the proximal coronary sinus with the insulated "tail" lying distally in the sinus-most clearly seen in the lateral view. 
a relatively large re-entry circuit and can often be terminated by single timed extrastimuli from several cardiac chambers. In contrast, the re-entry circuit in nodal tachycardia may be confined within or close to the atrioventricular node. An attempt to terminate such a tachycardia with single extrastimuli from conventional pacing sites is often frustrated by the distance from the re-entry circuit. Two or more precisely timed extrastimuli may be required to "peel back" the atrial or ventricular refractory period and invade the re-entry circuit. ${ }^{6}$ Sophisticated overdrive pacing systems have been designed $^{7}$ to overcome this problem but require reliable patient co-operation.

By using the closest practicable pacing site to the re-entry circuit, in the proximal coronary sinus, the best chance is obtained of stopping the tachycardia with a single extrastimulus, through a "window" (the period during the cycle when an extrastimulus will invade the re-entry circuit and stop the tachycardia) of maximal duration. This is an important consideration because dual demand underdrive pacers scan the cardiac cycle in a random fashion and the time taken for an impulse to fall in the critical window (and therefore terminate the tachycardia) will depend on the size of the window in relation to the cycle length of the tachycardia, and the rate of the pacemaker.

Advantages of dual demand pacing include automatic activation of the system, with no patient intervention needed, at the start of an arrhythmia, when there is the best chance of termination. ${ }^{4}$ Retention of pacing in bradycardia ensures a satisfactory rate after termination of tachycardia, especially where there is coexistent sinus node disease, and may prevent initiation of tachycardia by escape mechanisms. Low rate ( 70 a minute) atrial pacing is unlikely to start atrial fibrillation. The possibility of pacemaker activation by sinus tachycardia, starting nodal tachycardia, can be largely avoided if beta-blockers are used to depress sinus rate during exertion.

Although dual demand pacing is by no means suitable for all cases of refractory atrioventricular nodal tachycardia, in certain carefully selected cases it may provide a satisfactory and simple alternative to nodal ablation at open heart surgery with subsequent permanent ventricular pacing.

\section{References}

1 Ryan GP, Easley, RM Jr, Zarroff LI, Goldstein S. Paroxysmal use of a demand pacemaker in the treatment of supraventricular tachycardia due to the Wolff-Parkinson-White syndrome: observation on termination of reciprocal rhythm. Circulation 1968; 38: $1037-43$.

2 Dreifus LS, Arriaga J, Watanabe Y, Downing D, Haiat R, Morse D. Recurrent Wolff-Parkinson-White tachycardia in an infant. Am $\mathcal{F}$ Cardiol 1971; 28: 586-91.

3 Krikler D, Curry P, Buffet J. Dual-demand pacing for reciprocating atrioventricular tachycardia. $\mathrm{Br} \mathrm{Med}$ f 1976; i: $1114-6$.

4 Curry PVL, Rowland E, Krikler DM. Dual-demand pacing for refractory atrioventricular re-entry tachycardia. Pace 1979; 2: 137-51.

5 Curry PVL. Fundamentals of arrhythmias: modern methods of investigation. In: Krikler DM, Goodwin JF, eds. Cardiac arrhythmias, the modern electrophysiological approach. London: WB Saunders, 1975: 39-80.

6 Batchelor JE, Zipes DP. Treatment of tachyarrhythmias by pacing. Arch Intern Med 1975; 135: 1115-24.

7 Goyal SL, Lichstein E, Gupta PV, Chadda KD. Refractory re-entrant atrial tachycardia. $\mathrm{Am} \mathcal{f} \mathrm{Med}$ 1975; 58: 586-90.

Requests for reprints to Dr D B O'Keeffe, Department of Cardiology, Royal Victoria Hospital, Belfast, Northern Ireland. 